\title{
ANTI-INFLAMMATORY ACTIVITY OF NIGELLA SATIVA SILVER NANOPARTICLES: BIOCHEMICAL STUDY
}

\author{
GOMATHI KANNAYIRAM*, SANDHYA A, SOWMIYA S, VALARMATHI S, DANIEL JOSEPH \\ Department of Biotechnology, Dr. M.G.R Educational and Research Institute, University, Chennai - 600 095, Tamil Nadu, India. \\ Email: drgomathigopinathan@gmail.com
}

Received: 15 September 2018, Revised and Accepted: 30 October 2018

\section{ABSTRACT}

Objective: The aim of this study is to evaluate the anti-inflammatory activity of Nigella sativa silver nanoparticles (NS AgNPs).

Methods: Fourier transform infrared analysis was used to characterize the NS AgNPs and the extract. 2,2-diphenylpicrylhydrazyl assay was done to test the antioxidant potency of NS AgNP. Furthermore, in vitro anti-inflammatory activity of the extract and the NS AgNP was determined by red blood cell (RBC) membrane stabilization assay, protein inhibition assay, and interleukin-1 (IL-1) beta assay.

Results: The NS AgNP exhibited dose-dependent antioxidant property. At the concentration $0.01 \mathrm{mg} / \mathrm{ml} 80 \%$ of radical was scavenged by NS AgNP. Inhibition of protein denaturation assay also suggests that NS AgNP shows the highest activity (70\%) when compared with the standard drug aspirin (65\%). RBC assay suggests that NS AgNP stabilizes the RBC membrane and prevents leaking. In the enzyme-linked immunosorbent assay method the NS AgNP showed better IL-1 beta inhibition activity when compared to aqueous extract.

Conclusion: From the study, it was inferred that NS AgNPs are more effective when compared to the extract. These results suggest that NS AgNP can be used to treat inflammatory disorders.

Keywords: Nigella sativa, Fourier transform infrared, Nanoparticles, Protein denaturation, Enzyme-linked immunosorbent assay.

(C) 2019 The Authors. Published by Innovare Academic Sciences Pvt Ltd. This is an open access article under the CC BY license (http://creativecommons. org/licenses/by/4. 0/) DOI: http://dx.doi.org/10.22159/ajpcr.2019.v12i2.29775

\section{INTRODUCTION}

Inflammation is an important mechanism against various types of injury and infection. The inflammatory process is well defined and takes up two diverse phases such as acute and chronic. The accurate and appropriate emergence of acute inflammatory response against infection or injury are very essential [1].

Failure of the acute response in pathological condition leads to the uncontrolled chronic stimulation of inflammatory factors. Instead of being beneficial, stimulation of chronic inflammatory response leads to various undesirable physiological changes and paves the way for metabolic disorders such as arthritis, degenerative diseases, cancer, asthma, and atherosclerosis [2]. Due to this, there is an absolute need to reduce the chronic inflammation as a defensive mechanism to prevent the progression or emergence of metabolic disorders.

At present, steroidal and nonsteroidal drugs are preferred to prevent the pathological effects of the inflammatory response. Extended practice of these drugs forms the basis for the deterioration of tissues especially organs such as liver and kidney [3]. In this alarming situation, there is a need for alternative sources which could reduce the inflammatory response without triggering other pathological condition.

Various research approaches were being followed in the development of effective anti-inflammatory drugs from alternative sources such as medicinal plants and marine sources. Indian traditional medicinal system such as Siddha and Ayurveda uses various inorganic metals along with herbs to prevent and cure the chronic inflammatory pathological ailments [4]. Considering the above concept popular approach of bringing in the metal ions for the anti-inflammatory effect is possible only through the formation of nanoparticles [5,6]. In this regard, our study was based on the production of medical plant- based silver nanoparticles (AgNPs). For the production of AgNPs, we have used Nigella sativa (NS) as a medical plant. NS has been used extensively in Indian traditional medicines to cure various pathological conditions. Combination of metal ions and herbal medicine in the form of nanoparticle will produce beneficial effects by nullifying the problems associated with herbal formulation and metal ions [7]

\section{MATERIALS AND METHODS}

Collection of plant material

Seeds of NS (black cumin seeds) were collected and authenticated. The seeds were washed with water; shade dried, ground into a fine powder and kept in airtight container till use.

\section{Extraction from seeds}

Based on the protocol [8], the aqueous extract was prepared. Black cumin seeds were obtained from the local spice market. $30 \mathrm{~g}$ of black cumin seeds were ground to fine powder and soaked in distilled water in the ratio of 1:3 for 3 days with intermittent shaking. The extract was filtered using Whatman filter paper No.1 and was dried using China dish covered with perforated aluminum foil. The dried was stored at $4^{\circ} \mathrm{C}$ until further use.

\section{DETERMINATION OF ANTI-INFLAMMATORY ACTIVITY}

\section{Synthesis of NS AgNP's}

The synthesis procedure was carried based on the protocol [9]. $1 \mathrm{ml}$ of black cumin seeds aqueous extract was added to $10 \mathrm{ml}$ of $1 \mathrm{mM}$ silver nitrate solution with constant stirring for about $2 \mathrm{~h}$ at room temperature. The reaction mixture was checked periodically for color change. After $2 \mathrm{~h}$ of incubation time, the yellowish-green color was observed, which was then centrifuged at $3000 \mathrm{rpm}$ for $10 \mathrm{~min}$. The pellet contained the nanoparticles, which was separated and stored in a sterile Eppendrof's at $4^{\circ} \mathrm{C}$. 
Fourier transform infrared spectroscopy (FTIR)

The liquid form of plant extract and the synthesized AgNP's was used for performing FTIR analysis using Perkin Elmer spectrum 1: FTIR spectrometer with a scan range of $450-4000 \mathrm{~cm}^{-1}$ and with the resolution of $1.0 \mathrm{~cm}^{-1}$.

\section{Scanning electron microscope (SEM)}

The NS extract and the black cumin seeds AgNP's were diluted using buffer solution were determined to study the morphology of AgNPs and the extract. The images were taken using the SEM imaging mode at a scan frequency of $0.5 \mathrm{~Hz}$.

\section{2,2-diphenylpicrylhydrazyl (DPPH) radical scavenging method}

Based on the protocol [9], 0.1 mM DPPH solution of was prepared using methanol and $100 \mu \mathrm{l}$ of DPPH was added to the extract solutions of different concentrations ( $5 \mathrm{mg}$ of extract is mixed with $2.5 \mathrm{ml}$ of ethanol). The mixture was shaken vigorously and allowed to stand at room temperature for $30 \mathrm{~min}$. The absorbance was measured at $517 \mathrm{~nm}$ using a ultraviolet-visible (UV-Vis) spectrophotometer. DPPH alone serves as a control.

\section{Red blood cell (RBC) membrane stabilization assay}

Based on the protocol [9], $2 \mathrm{ml}$ of blood was drawn and added to EDTA to prevent coagulation. To $0.5 \mathrm{ml}$ of $\mathrm{RBC}$ different concentrations of the NS extract and the synthesized NS AgNP are added along with
Triton $\mathrm{x} 100$. The mixture was incubated at $37^{\circ} \mathrm{C}$ for $30 \mathrm{~min}$. $\mathrm{RBC}$ with triton $\times 100$ water serves as positive control. RBC without extract, NS AgNP,and triton $\times 100$ act as negative control the sample mixture was then centrifuged at $300 \mathrm{rpm}$ for $10 \mathrm{~min}$. The supernatant was removed from each tube and analyzed for the stabilization. The absorbance was measured using UV-Vis spectrophotometer at $517 \mathrm{~nm}$. The triplicates values obtained were calculated.

\section{PROTEIN DENATURATION}

To the reaction mixture of bovine serum albumin, NS extract and the synthesizeed NS AgNP are added in different concentrations. The mixture was incubated at $37^{\circ} \mathrm{C}$ for $20 \mathrm{~min}$ followed by heating at $70^{\circ} \mathrm{C}$ for $10 \mathrm{~min}$. After cooling, the turbidity was measured at $600 \mathrm{~nm}$ [10].

\section{Enzyme-linked immunosorbent assay (ELISA)}

Interleukin-1 (IL-1) beta ( $\beta$ ) was analyzed from lymphocyte cell culture supernatants by the help of commercially available ELISA kit (bioassay technology) according to the protocol advised along with the kit. All samples measured in triplicates [11].

\section{Statistical analysis}

All the data were analyzed using statistical software Minitab. Data were expressed as the mean \pm standard deviation using ANOVA and t-test. $\mathrm{p}$ values are to be statistically significant with $<0.05$.

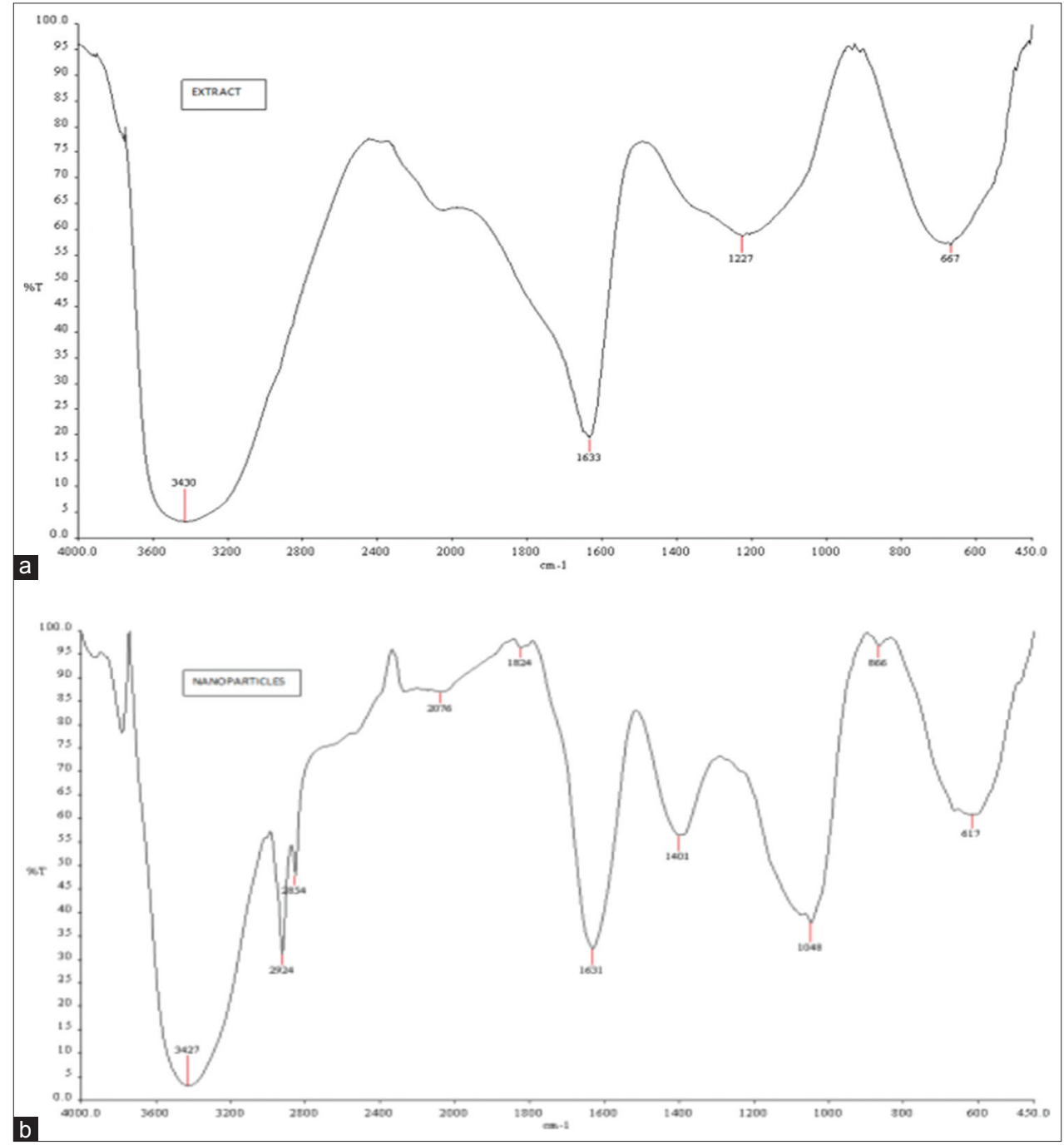

Fig. 1: (a and b) Fourier transform infrared spectrum of Nigella sativa (NS) aqueous extract and NS silver nanoparticles 


\section{RESULTS AND DISCUSSION}

Standard anti-inflammatory drug causes deleterious effects which take the road to go for development of harmless substitutes [12]. Such an alternative is plant-based medicines. Due to their instability, it is not being chosen as ideal for therapeutic. To enhance the excellence of plant-based medicine, the traditional approach such as Siddha and Ayurveda has used inorganic metallic ions along with herbs. By taking the essence of Siddha and Ayurveda, in this study, we have synthesized NS AgNP and analyzed the anti-inflammatory activity.

\section{FTIR analysis}

The FTIR spectrum is used to determine the various functional groups, which is a capping agent, Fig. 1. The synthesized nanoparticles exhibit a peak at $1824 \mathrm{~cm}^{-1}$ and $886 \mathrm{~cm}^{-1}$ represents $\mathrm{N}-\mathrm{H}$ stretching while the bands observed at $1631 \mathrm{~cm}^{-1}$ and $1401 \mathrm{~cm}^{-1}$ represents $\mathrm{C}=0$ stretching.

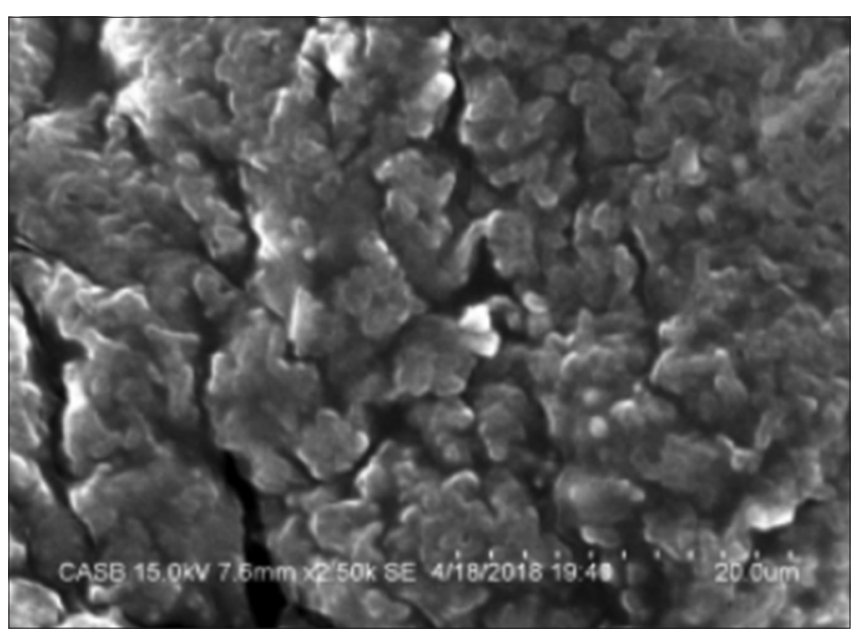

Fig. 2: Scanning electron microscope image of silver nanoparticles

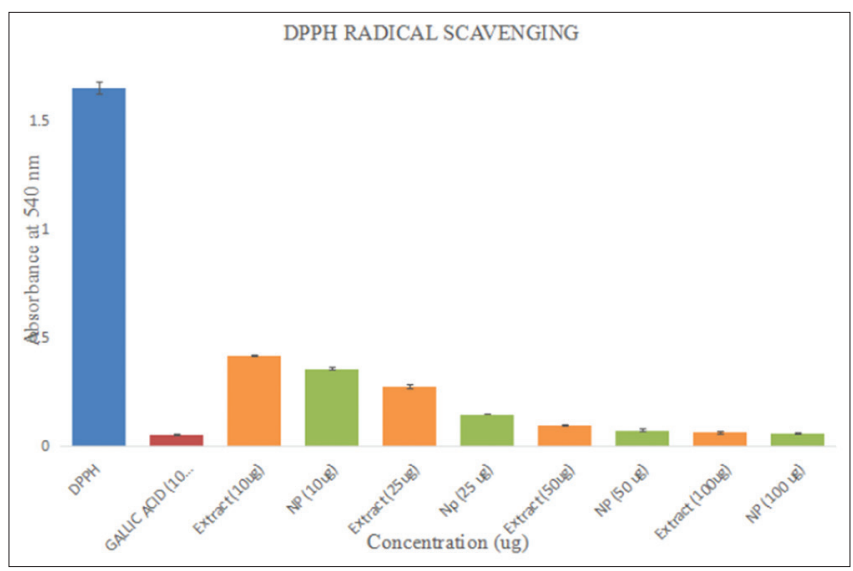

Fig. 3: Antioxidant activity of Nigella sativa (NS) silver nanoparticles and the NS extract along with control
The $\mathrm{N}=0$ peaks are at $1048 \mathrm{~cm}^{-1}$ and $2924 \mathrm{~cm}^{-1}$. The smaller peaks represent the alkynes. Similarly, the ethyl acetate extract exhibits peak at $1227 \mathrm{~cm}^{-1}$ indicating the $\mathrm{N}-\mathrm{H}$ stretching. The amide band of $\mathrm{C}=\mathrm{O}$ stretching is indicated at $1633 \mathrm{~cm}^{-1}$. From this analysis, it is clear that the AgNP exhibit higher peaks through capping with the functional groups.

\section{SEM analysis}

The synthesized AgNPs were cuboidal in shape and without aggregation when compared to the extract, as shown in Fig. 2. The range is between $20 \mathrm{~nm}$ and $100 \mathrm{~nm}$ in size. These variations in size and shape occur during the synthesis of AgNP.

\section{DPPH radical scavenging (antioxidant activity)}

Several research findings suggest that there is a close connection of generation reactive oxygen species (ROS) with inflammation [13]. During the inflammatory process, ROS is generated as a defensive mechanism in chronic inflammation. Besides the beneficial effects, the uncontrolled synthesis of ROS leads to deleterious effect [14]. In this regard, NS AgNP were analyzed for its antioxidant activity. Both NS extract and the NS AgNP show better antioxidant activity. In spite of this, NS AgNP showed better activity when compared to the NS extract. Fig. 3: From Figure 3, it was inferred that both the extract and NS AgNP showed dose-dependent scavenging activity. Even at the lower concentration $(0.01 \mathrm{mg} / \mathrm{ml})$, NS AgNP's possessed high radical scavenging activity almost $80 \%$ radicals were scavenged from the data it was incidental that NS AgNP's have higher antioxidant potency.

\section{RBC membrane stabilization assay}

Prevention of hemolysis by the NS AgNP's and extract forms the basis for this assay. Triton $\mathrm{x} 100$ being detergent destabilizes the RBC membrane and allows leaking of hemoglobin. Three different concentrations of the extract and NS AgNP's were analyzed. The results clearly suggest that NS AgNP's and extracts prevent the leaking of hemoglobin by stabilizing the RBC structure and protects the integrity of RBC. Indirectly this study also suggests that NS AgNP's are nontoxic in nature and can be used for further biological studies (Fig. 4).

\section{Protein denaturation}

Denaturation of proteins is one of the deleterious effects of inflammation and paves the way for various degenerative disorders [15]. During the inflammatory process, protein lose their complex structure and gets aggregated and bigger. On this basis, a NS AgNP's and extract were studied for its role in the inhibition of denaturation of the protein. From Figure 5, it was depicted that AgNP prevented the aggregation of the protein (Fig. 5).

\section{ELISA}

An marked increased in level of IL-1 $\beta$ cytokine was experienced in various metabolic disorders, and bacterial infections inhibition of these cytokine might help in preventing the advancement of pathogenic effects of the metabolic disorders [16]. In this observe, downregulation of this cytokine is an essential pharmacological goal for inflammatory based disorders. The NS AgNP's and extract in inhibition of IL-1 $\beta$ were analyzed and compared. The effect of the extract and the AgNPs was expressed in Fig. 6. It is evident that the NS AgNP's shown to be highly potent in the inhibition of IL- $1 \beta$ expression when compared to the extract. The samples are also compared with the standard IL- $1 \beta$, and the hydrogen peroxide treated cells. Since the NS AgNP's exhibited the

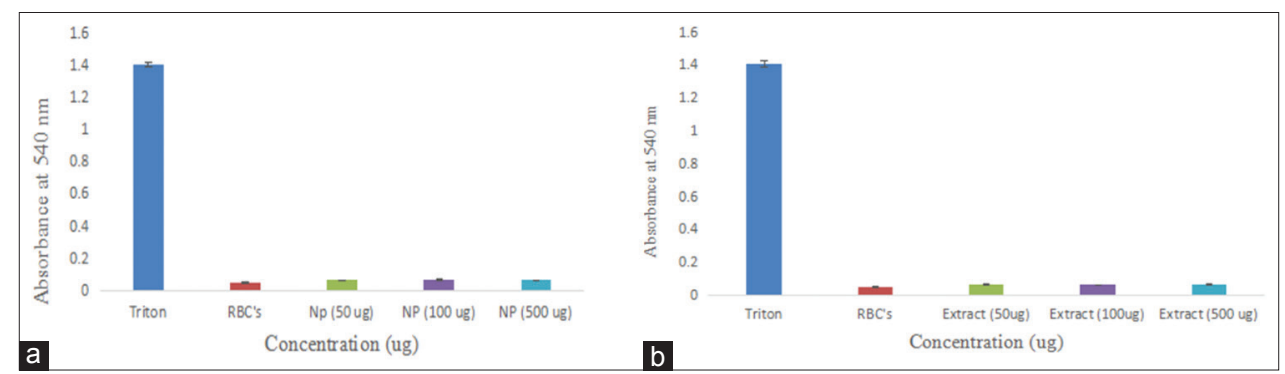

Fig. 4: (a and b) Red blood cell membrane stabilization activity of Nigella sativa (NS) silver nanoparticles and the NS extract along with control 


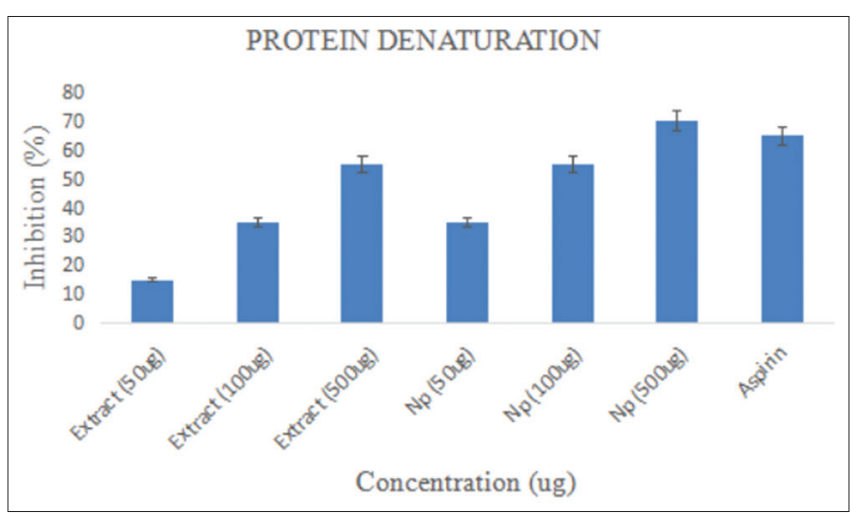

Fig. 5: Proteolytic activity of silver nanoparticles (AgNPs) and Nigella sativa extract. AgNPs showed maximum inhibition

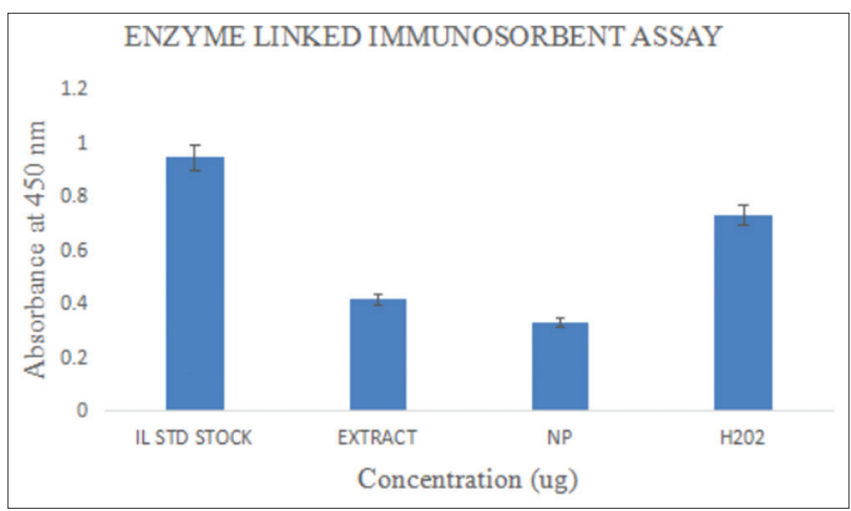

Fig. 6: Interleukin-1 beta inhibition assay by the Nigella sativa (NS) silver nanoparticles and the extract

effect of inhibition at a lower concentration, the results revealed that it has anti-inflammatory property.

\section{CONCLUSION}

From the above study, it was inferred that NS AgNP's possessed high antioxidant and anti-inflammatory activity when compared to the aqueous extract of NS. These results suggest that it can be used as a therapy for inflammatory disorders.

\section{ACKNOWLEDGMENTS}

We thank DR. M.G.R. Educational and Research Institute for the constant encouragement and support through their infrastructure and research facilities.

\section{AUTHORS' CONTRIBUTION}

Gomathi Kannayiram - High contribution. Sandhya A - Medium contribution. Other authors - Low and equally contributed.

\section{CONFLICTS OF INTEREST}

None of the authors have a conflict of interest in this manuscript.

\section{REFERENCES}

1. Baizabal-Aguirre VM, Rosales C, López-Macías C, Gómez MI. Control and resolution mechanisms of the inflammatory response 2016. Mediators Inflamm 2016;2016:3591797.

2. Raghavan R, Cheriyamundath S, Madassery J. Exploring the mechanisms of cytotoxic and anti-inflammatory property of andrographolide and its derivates. Pharmacogn Rev 2016;12:56-65.

3. Verma S. Medicinal plants with anti-inflammatory activity. J Phytopharmacol 2016;5:157-9.

4. Ghasemian M, Owali S, Owlia MB. Review of anti-inflammatory herbal medicines. Adv Pharmacol Sci 2016;2016:1-11.

5. Al-Ghamdi MS. The anti-inflammatory, analgesic and antipyretic activity of Nigella sativa. J Ethnopharmacol 2001;76:45-8.

6. Deepa M, Renuka D. Potential anti-inflmmatory medicinal plants-a review. Int J Pharm Pharm Sci 2014;6:43-9.

7. Rakesh W, Saranya S, Gurukarthikeyan, Kumar PR, Lokeshmari SS, Gayathri D, et al. Chitosan gallic acid microsphere incorporated collagen matrix for chronic wounds: Biophysical and biochemical characterization. Int J Pharm Pharm Sci 2014;6:94-100.

8. Mary EJ, Inbathamizh L. Green synthesis and characterization of nano silver using leaf extract of Morinda pubescens. Asian J Pharm Clin Res 2012;5:159-62.

9. Varghese RE, Raghavan D, Sivaraj S, Gayathri D, Kannayiram G. Antiinflammatory activity of Syzgium aromaticum silver nanoparticles: In vitro and in silico study. Asian J Pharm Clin Res 2017;10:370-3.

10. Farid R, Day T, Friesner RA, Pearlstein RA. New insights about HERG blockade obtained from protein modeling, potential energy mapping, and docking studies. Bioorg Med Chem 2006;14:3160-73.

11. Quillin ML, Wingfield PT, Matthews BW. Determination of solvent content in cavities in IL-1beta using experimentally phased electron density. Proc Natl Acad Sci U S A 2006;103:19749-53.

12. Leelaprakash G, Dass SM. In vitro anti-inflammatory activity of methanol extract of Enicostemma axillare. Int J Drug Dev Res 2011;3:189-96

13. Pisoschi AM, Negulescu GP. Methods for total antioxidant activity determination: A review. Biochem Anal Biochem 2011;1:106.

14. Ansari AQ, Ahmed SA, Waheed MA, Juned AS. Extraction and determination of antioxidant activity of Withania somnifera Dunal. Eur J Exp Biol 2013;3:502-7.

15. Neurath $\mathrm{H}$. Proteolytic processing and physiological regulation. Trends Biochem Sci 1989;14:268-71.

16. Bang JS, Oh DH, Choi HM, Sur BJ, Lim SJ, Kim JY, et al. Antiinflammatory and antiarthritic effects of piperine in human interleukin 1beta-stimulated fibroblast-like synoviocytes and in rat arthritis models. Arthritis Res Ther 2009;11:R49. 